\title{
Urinary levels of podocyte-derived microparticles are associated with the progression of chronic kidney disease
}

\author{
Jian Lu, Ze-Bo Hu, Pei-Pei Chen, Chen-Chen Lu, Jia-Xiu Zhang, Xue-Qi Li, Ben-Yin Yuan, \\ Si-Jia Huang, Kun-Ling Ma
}

Institute of Nephrology, Zhongda Hospital, School of Medicine, Southeast University, Nanjing 210009, China

Contributions: (I) Conception and design: KL Ma; (II) Administrative support: KL Ma; (III) Provision of study materials or patients: J Lu; (IV) Collection and assembly of data: J Lu; (V) Data analysis and interpretation: J Lu; (VI) Manuscript writing: All authors; (VII) Final approval of manuscript: All authors.

Correspondence to: Kun-Ling Ma, MD, PhD. Institute of Nephrology, Zhongda Hospital, School of Medicine, Southeast University, No. 87 Ding Jia Qiao Road, Nanjing 210009, China. Email: klma05@163.com.

\begin{abstract}
Background: Podocyte-derived microparticles (MPs) could be secreted from activated or apoptotic podocytes. An increased number of podocyte-derived MPs in the urine might reflect podocyte injury in renal diseases. This study aimed to observe the change of urinary podocyte-derived MP levels in patients with chronic kidney disease (CKD) and to further explore its correlation with the progression of CKD.

Methods: A prospective, longitudinal study was conducted in eighty patients with biopsy-proven CKD. Podocyte-derived MPs (annexin V and podocalyxin positive) were detected by flow cytometry. The number of urinary podocyte-derived MPs was analyzed to evaluate the association with biochemical measurements and pathological glomerulosclerosis assessment. Patients with idiopathic membranous nephropathy (IMN) were followed up after the six-month treatment of prednisone combined with tacrolimus to evaluate the association of urinary podocyte-derived MP levels and the remission of IMN.

Results: The CKD patients had higher urinary podocyte-derived MP levels compared with healthy controls (HCs). Baseline urinary levels of podocyte-derived MPs were positively correlated with 24-hour proteinuria, while were inversely correlated with the percentage of global glomerulosclerosis. The urinary podocyte-derived MPs levels had good discrimination for glomerulosclerosis [area under curve (AUC), 0.66]. The urinary podocyte-derived MPs levels in IMN patients were significantly decreased accompanied with the recovery of abnormal clinical parameters after six-month treatment.

Conclusions: The urinary levels of podocyte-derived MPs were closely associated with podocyte injury and glomerulosclerosis, which could be useful for monitoring disease activity in CKD patients. Urinary podocyte-derived MPs might be a non-invasive biomarker for the evaluation of early CKD progression.
\end{abstract}

Keywords: Podocyte-derived microparticle (podocyte-derived MP); podocyte injury; chronic kidney disease $(\mathrm{CKD})$

Submitted Jun 22, 2019. Accepted for publication Aug 13, 2019.

doi: $10.21037 /$ atm.2019.08.78

View this article at: http://dx.doi.org/10.21037/atm.2019.08.78

\section{Introduction}

Glomerulosclerosis is pathological during chronic kidney disease (CKD) progressed to end-stage renal disease (ESRD) (1). Although renal biopsy is the "gold standard" diagnosis method for glomerular diseases, it is traumatic and difficult for clinical repetition. Therefore, the early detection of noninvasive biomarkers related to the process of cytological change is likely to be novel strategies for early monitoring of renal lesions in the development of CKD.

Podocyte injury is a crucial cytological event in the 
pathogenesis and progression of CKD (2). Podocytes are the major components for maintaining structural integrity of glomerular filtration barrier (GFB) and achieving selective filtration. Podocytes are non-replicating cells, and all kinds of mechanical stress (3), oxidative stress (4) and immune disorder (5) can cause podocyte foot process fusion, podocyte detachment, eventually leading to proteinuria, glomerulosclerosis, and renal function decline (6). Therefore, early detection of podocyte injury might contribute to early intervention and prevent the progression of CKD.

Urine is the main source of biomarkers for developing new diagnostic tools to identify and stratify CKD patients. Urine may be a non-invasive "window" for the insight into physiological status of the whole renal system. Recently, some studies suggest that urine microparticles (MPs) possess potential diagnosis application values for renal diseases (7). The MPs, a subtype of extracellular vesicles (EVs), are a heterogeneous group that are secreted from activated or apoptotic cells, ranging at 100-1,000 nm in diameter (8). The MPs are global structures with bilayer membrane containing bioactive molecules (such as proteins, microRNAs, and RNAs) and thereby serve as vectors for intercellular interaction (9). Recent studies suggest that urinary podocyte-derived MPs may be correlated with the degree of podocyte damage, or the disease activity of glomerulonephritis, as the urinary levels of podocyte-derived MPs are increased before existence of microalbuminuria at the early stage of diabetic nephropathy (DN) in mice models (10). In renovascular hypertensive patients, there is an elevated ratio of urinary podocin ${ }^{+} \mathrm{EVs}$ to nephrin ${ }^{+} \mathrm{EVs}$ (11).

Therefore, this study aimed to investigate the change of urinary podocyte-derived MP levels in CKD patients and further explore its correlation with the progression of CKD.

\section{Methods}

\section{Patients}

This prospective study was conducted at Department of Nephrology, Zhongda Hospital, Southeast University, China, between October 2015 and October 2017. Eighty CKD patients [idiopathic membranous nephropathy (IMN), $\mathrm{n}=27$; $\operatorname{IgA}$ nephropathy (IgAN), $\mathrm{n}=19$; lupus nephritis (LN), $\mathrm{n}=17$; DN, $\mathrm{n}=17$ ] were newly diagnosed and enrolled before initial treatments. The enrolled patients with IMN were treated with prednisone plus tacrolimus. Prednisone was given orally at $1 \mathrm{mg} / \mathrm{kg} / \mathrm{d}$ and the plasma concentration of tacrolimus was controlled at $5-10 \mathrm{ng} / \mathrm{mL}$. There were only $10 \mathrm{IMN}$ patients with complete follow-up data as the subjects were lost to follow up. None of them received kidney transplantation. The blood and urine samples from 10 IMN patients were collected again after 6-month immunosuppressive therapy.

\section{Renal histopathological assessment}

Histopathologic assessment of renal biopsy specimens was performed with light microscopy, immunofluorescent staining, and electron microscopy. The score of glomerulosclerosis severity was assessed semi-quantitatively by two nephropathologists blinded to the laboratory. Global glomerulosclerosis was defined as scarring lesion or hyaline deposition in more than half of the glomerulus. Global glomerulosclerosis was scored according to the percentage of global glomerulosclerosis in whole renal biopsy specimens from each patient.

\section{Clinical and biochemical data}

The clinical and pathological data were collected at the time of enrollment for all CKD patients. Biochemical parameters were obtained from clinical data.

\section{Isolation and analysis of MPs}

MPs isolation from urine samples by differential centrifugation was as described (12). After collecting $50 \mathrm{~mL}$ spot morning urine for every participant, protease inhibitors cocktail was added to urine sample and all urine samples were centrifuged at $3,000 \mathrm{~g}$ for 10 minutes at $18^{\circ} \mathrm{C}$. The supernatants were then centrifuged at $18,000 \mathrm{~g}$ for 20 minutes at $18{ }^{\circ} \mathrm{C}$. Pelleted MPs were resuspended with annexin $V$ binding buffer. The MPs were quantified using a flow cytometer controlled by Cell Quest Pro 4.0.2 software (BD Bioscience, USA). For MPs labeling, an allophycocyanin-labeled annexin $\mathrm{V}$ antibody was used for identifying events as total MPs. A phycoerythrin (PE)-conjugated podocalyxin antibody (eBioscience, USA) was employed to identify podocyte origin. MPs were defined as particles ranging at $0.1-1 \mu \mathrm{m}$ in diameter and exhibited positive specific fluorescence compared with negative controls.

According to the manufacturer's instructions, the gate strategy for the MP detection was as following: compensation for channel spill was calculated using the auto-compensation feature from recorded values of 
Table 1 Clinical characteristics of HCs and CKD patients

\begin{tabular}{|c|c|c|c|}
\hline Parameter & HCs $(n=30)$ & CKD $(n=80)$ & $P$ value \\
\hline Sex (female/male) & $10 / 20$ & $34 / 46$ & $<0.001$ \\
\hline SBP $[\mathrm{mmHg}]$ & $118[110-127]$ & $140[129-156]$ & $<0.001$ \\
\hline $\mathrm{DBP}[\mathrm{mmHg}]$ & 82 [70-89] & 85 [75-92] & 0.15 \\
\hline Fast glucose (mmol/L) & $5.90 \pm 0.90$ & $5.43 \pm 0.53$ & 0.18 \\
\hline Total cholesterol (mmol/L) & $4.11 \pm 2.28$ & $6.60 \pm 2.37$ & $<0.001$ \\
\hline Triglyceride (mmol/L) & $2.19(1.41,3.05)$ & $2.49(1.35,4.35)$ & 0.06 \\
\hline HDL-cholesterol (mmol/L) & $1.71 \pm 0.67$ & $1.43 \pm 0.44$ & 0.05 \\
\hline BUN (mmol/L) & $4.40(3.70,5.00)$ & $5.49(4.43,8.55)$ & 0.001 \\
\hline Serum creatinine $(\mu \mathrm{mol} / \mathrm{L})$ & $80.0(71.0,84.0)$ & $82.50(69.25,109.50)$ & 0.31 \\
\hline Serum uric acid (mmol/L) & $340.84 \pm 76.51$ & $377.35 \pm 109.53$ & 0.10 \\
\hline Proteinuria (g/24 hours) & - & $4.50(2.63,6.63)$ & - \\
\hline eGFR $\left(\mathrm{mL} / \mathrm{min}\right.$ per $\left.1.73 \mathrm{~m}^{2}\right)$ & $92.57(82.79,101.47)$ & $87.17(65.85,106.72)$ & 0.27 \\
\hline ARB/ACEI & 0 & $37 / 43$ & - \\
\hline
\end{tabular}

Results are expressed as means \pm SD or medians (IQRs). P value, CKD vs. HCs. HCs, healthy controls; CKD, chronic kidney disease; SBP, systolic blood pressure; DBP, diastolic blood pressure; HDL, high-density lipoprotein; LDL, low-density lipoprotein; BUN, blood urea nitrogen; eGFR, estimated glomerular filtration rate; ARB, angiotensin receptor blocker; ACEl, angiotensin converting enzyme inhibitors; $\mathrm{SD}$, standard deviation; IQRs, interquartile ranges.

separate and combined unstained and single-stained MP. The thresholds were set with isotype control fluorescent antibodies. MP prepared and stained in only annexin $\mathrm{V}$ binding buffer without calcium served as negative controls. Before analyzing the formal samples, the gate setting for MP detection was conducted. Standard beads of $0.8 \mu \mathrm{m}$ (P1 gate) in diameter were used for size calibration and helped to set the MPs gate. To provide the absolute count of MPs in a sample, standard beads (P2 gate) of $3 \mu \mathrm{m}$ in size for a known concentration were used as internal standard, the beads were added to each sample before measurement. The absolute number of MPs was calculated as following equation: (MPs counting $\times$ standard $3 \mu \mathrm{m}$ beads $/ \mathrm{L}) /$ standard $3 \mu \mathrm{m}$ beads counting. Data are described as $\times 10^{6}$ events $/ \mathrm{mL}$ of urine.

\section{Statistical analysis}

Data analysis was conducted using SPSS Version 20.0 (IBM SPSS Statistics, USA). Quantitative variables were summarized as means \pm standard deviation (SD) or median with interquartile range (IQR) for appropriate. Intergroup comparisons were analyzed with the Kruskal-Wallis test and the chi-squared test. Bivariate correlation was performed by Spearman's rank correlation. The receiveroperating characteristic (ROC) curve was used to evaluate the discrimination value of MPs. The significance level was judged at $\mathrm{P}<0.05$.

\section{Results}

\section{Demographic data}

The clinical and laboratory features of subjects were summarized in Table 1. The CKD group $(\mathrm{n}=80)$ comprised of $\operatorname{IMN}(\mathrm{n}=27), \operatorname{IgAN}(\mathrm{n}=19), \mathrm{LN}(\mathrm{n}=17)$ and $\mathrm{DN}(\mathrm{n}=17)$. Thirty-six patients $(42.5 \%)$ were female and mean age was $46.58 \pm 13.70$ years. Thirty healthy controls (HCs, 10 females) with mean age $41.77 \pm 9.27$ years were recruited. 
A

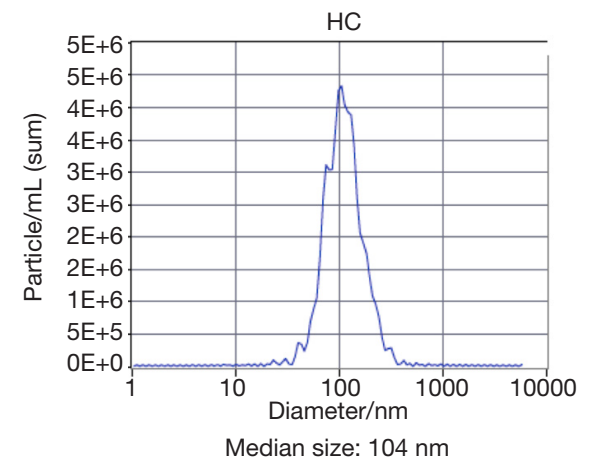

B

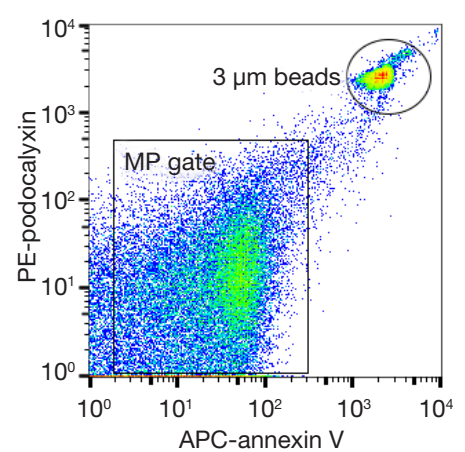

C

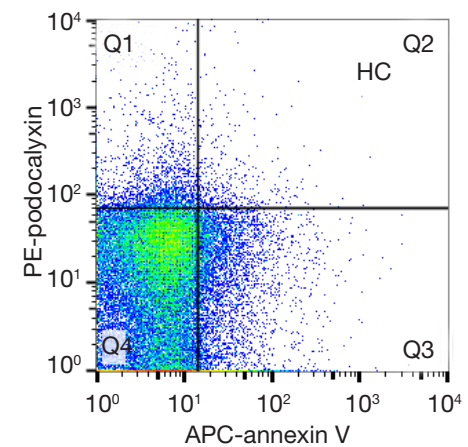

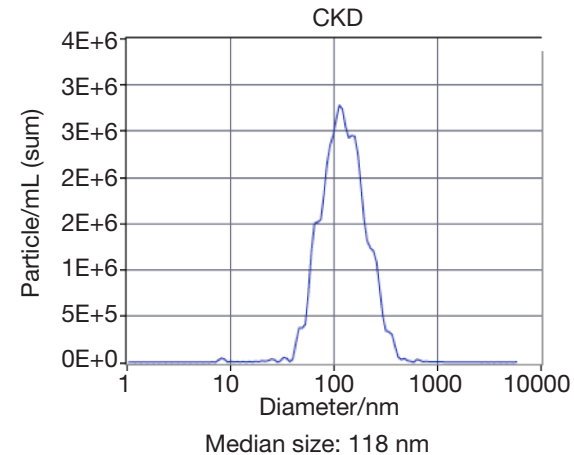

Median size: $118 \mathrm{~nm}$

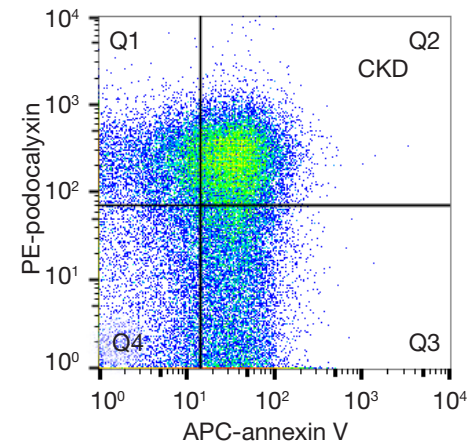

Figure 1 Urinary levels of annexin $\mathrm{V}$ and podocalyxin positive MPs are elevated in CKD patients. (A) NTA showed the distribution and median size of EVs derived from urine samples from HCs and CKD patients; (B,C,D) representative flow cytometry scatter plots of annexin $\mathrm{V}$ and podocalyxin positive MPs (defined as podocyte-derived MPs) in HCs and CKD patients. CKD, chronic kidney disease; NTA, nanoparticles tracking analysis; EVs, extracellular vesicles; HCs, healthy controls; MPs, microparticles; PE, phycoerythrin; APC, allophycocyanin.

Baseline data showed that systolic blood pressure (SBP), serum total cholesterol, low-density lipoprotein (LDL)cholesterol, LP(a) and blood urea nitrogen (BUN) in CKD patients were significantly higher than that in HCs, while serum albumin level was lower than that in HCs. There was no difference for the serum creatinine and estimated glomerular filtration rate (eGFR) compared CKD patients with HCs.

\section{Urinary levels of annexin $V$ and podocalyxin positive MPs in CKD patients}

Nanoparticles tracking analysis (NTA) confirmed the distribution and median size of EVs derived from urine samples from HCs and CKD patients (Figure 1A). Absolute numbers of annexin $\mathrm{V}$ and podocalyxin positive MPs (defined as podocyte-derived MPs) in urine samples from all subjects were quantified by flow cytometry (Figure 1B,C,D).

The levels of urinary podocyte-derived MPs were summarized in Table 2. Compared with HCs, there was significant increase of urinary podocyte-derived MPs in
CKD patients (Figure $2 A, B$ ). Furthermore, urinary levels of podocyte-derived MPs were significantly different among CKD patients with different levels of proteinuria (Figure 2C). We compared the podocyte-derived MP levels among CKD patients with different degrees of glomerulosclerosis and tubulointerstitial lesions, separately. The CKD patients with glomerulosclerosis showed lower urinary levels of podocyte-derived MPs compared with those without glomerulosclerosis $(\mathrm{P}<0.05)$ (Figure $2 D)$. The patients with global glomerulosclerosis showed lower urinary levels of podocyte-derived MPs compared with those without global glomerulosclerosis (Figure 2E). Urinary levels of podocytederived MPs were not significantly different among CKD patients with different degree of tubulointerstitial lesions (Figure 2F).

\section{Correlation analysis of urinary podocyte-derived MPs levels with clinical parameters of CKD patients}

Furthermore, we analyzed the possible correlation between clinical parameters and urinary levels of podocyte-derived 
Table 2 The levels of urinary podocalyxin positive MPs in HCs and the patients

\begin{tabular}{lcc}
\hline Groups & Numbers & The levels of urinary podocalyxin positive $\mathrm{MPs}\left(10^{6} / \mathrm{mL}\right.$ urine $)$ \\
\hline HCs & 30 & $1.05(0.46,2.77)$ \\
IMN & 27 & $4.87(3.91,7.34)$ \\
IgAN & 19 & $2.51(1.08,5.50)$ \\
LN & 17 & $2.73(2.29,3.95)$ \\
DN & 17 & $3.55(1.80,6.67)$ \\
\hline
\end{tabular}

Results of urinary podocalyxin positive MPs are expressed as medians (IQRs). HCs, healthy controls; MPs, microparticles; IMN, idiopathic membranous nephropathy; IgAN, IgA nephropathy; LN, lupus nephritis; DN, diabetic nephropathy; IQRs, interquartile ranges.

MPs in patients with CKD. We found a significant correlation between the urinary levels of podocyte-derived MPs and serum levels of high-density lipoprotein (HDL)-cholesterol, LDL-cholesterol, 24-hour proteinuria and urinary $\mathrm{N}$-acetylbeta-glucosaminidase (NAG). Urinary levels of podocytederived MPs were negatively associated with serum albumin levels. However, no significant association was detected between the urinary levels of the podocyte-derived MPs and age, SBP, serum levels of total cholesterol, triglyceride, and creatinine or eGFR respectively (Figure 3).

\section{Assessment on the discrimination power of urinary podocyte-derived MP levels for disease activities}

As shown in ROC curves (Figure 4), urinary podocytederived MP levels differentiated CKD patients with HCs [area under curve (AUC), 0.82] $(13,14)$. Urinary podocytederived MP levels differentiated CKD patients with global glomerulosclerosis or without global glomerulosclerosis (AUC, 0.66).

\section{Correlation analysis of urinary podocyte MP levels with global glomerulosclerosis in CKD patients}

Univariate associations of podocyte-derived MP levels with global glomerulosclerosis in CKD patients were given in Table 3. Global glomerulosclerosis was positively associated with SBP, serum glucose, BUN, and serum creatinine, and negatively associated with serum total cholesterol, serum LDLcholesterol, eGFR and urinary levels of podocyte-derived MPs.

\section{Effect of immunosuppressive therapy on the urinary levels of podocyte MPs in patients with IMN}

Then, we analyzed the possible influence of immunosuppressive therapy of prednisone combined with tacrolimus on the urinary levels of podocyte-derived MPs in IMN patients. A total of ten IMN patients were studied before and after the administration of immunosuppressive therapy. We observed a significant decrease of the urinary levels of podocyte-derived MPs as proteinuria declined after six-month therapy (Figure 5).

\section{Discussion}

This study showed that the levels of annexin $\mathrm{V}$ and podocalyxin positive MPs were significantly higher in CKD patients compared with those in HCs. The diagnosis of the patients enrolled in our research was based on the pathological evaluation of the kidney tissues. In the primary or secondary glomerular diseases, podocyte injuries often occur with varying degrees. Podocyte derived MPs shedding could be common pathophysiologic changes during the disease progression, which can provide a new aspect for the evaluation of podocyte injuries.

It's well-known that podocalyxin is a transmembrane protein mainly expressed on the apical membrane of glomerular podocytes. In addition, podocalyxin is also expressed on vascular endothelium, hematopoietic progenitor cells, and some certain neurons (13-15). Structurally, podocalyxin acts as the main contributor to the anionic surface of glomerular podocyte foot processes, involved in the normal formation and maintenance of podocyte structure through its connection with the actin cytoskeleton (16). Previous studies demonstrated that there were elevated urinary levels of podocalyxin protein in patients with diabetes and other glomerular diseases as determined by enzyme linked immunosorbent assay (ELISA) (17). In this study, using flow cytometer assay, we examined the relative quantification and distribution of podocyte-derived MPs labelled by annexin $\mathrm{V}$ and podocalyxin, which was different from the urinary excretion 
A

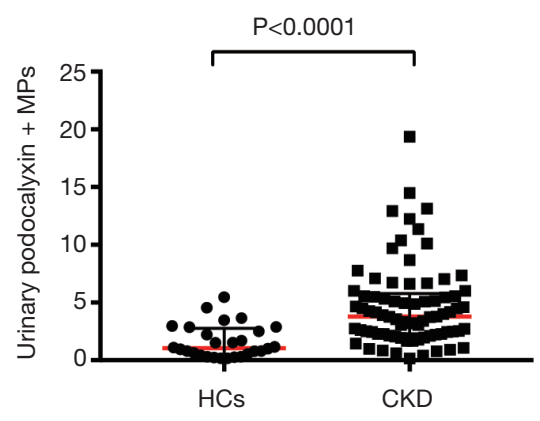

C

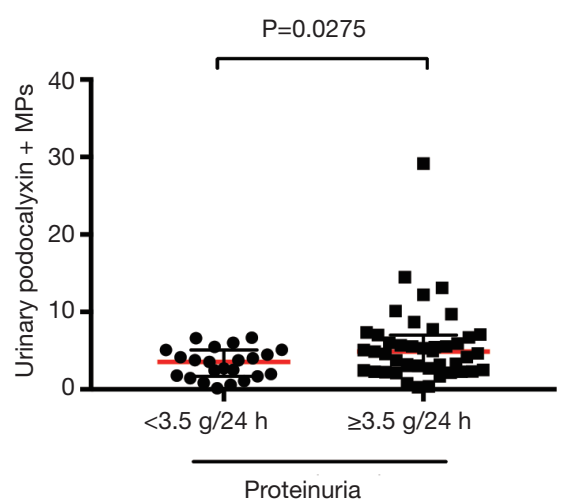

$\mathrm{E}$

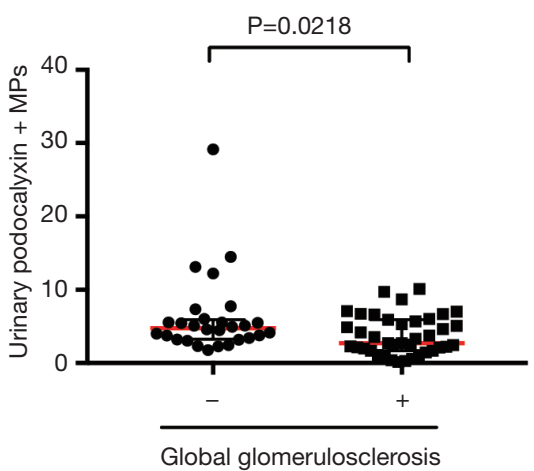

B

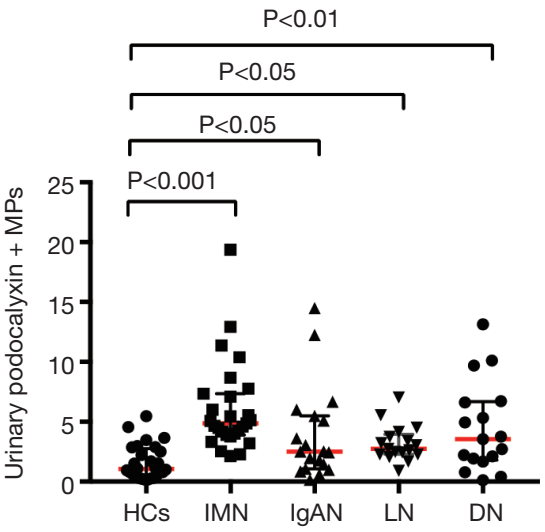

D

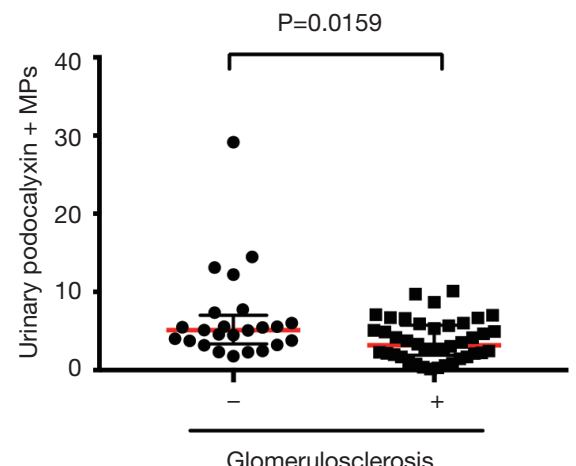

$\mathrm{F}$

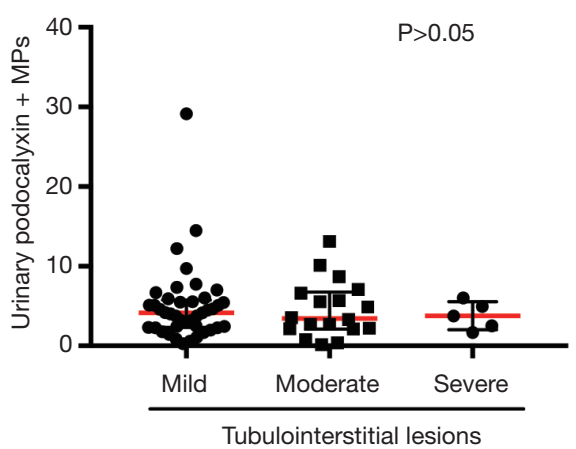

Figure 2 Urinary levels of podocyte-derived MPs were elevated in biopsy-proven CKD patients. (A) Comparison of the levels of urinary podocyte-derived MPs from CKD patients and HCs; (B) scatter dot plots showed the difference in the numbers of urinary podocytederived MPs in different pathological types of CKD (IMN, n=27; IgAN, n=19; LN, n=17; DN, n=17); (C) scatter dot plots showed urinary levels of podocyte-derived MPs according to the different degree of tubulointerstitial lesions (mild, n=43; moderate, $n=18$; severe, $n=5$ ); (D) scatter dot plots showed urinary levels of podocyte-derived MPs according to the presence of glomerulosclerosis (no glomerulosclerosis, $\mathrm{n}=26$; glomerulosclerosis, $\mathrm{n}=40$ ); (E) scatter dot plots showed urinary levels of podocyte-derived MPs according to the presence of global glomerulosclerosis (no global glomerulosclerosis, $n=28$; global glomerulosclerosis, $n=38$ ); (F) scatter dot plots showed urinary levels of annexin $\mathrm{V}$ and podocalyxin-positive MPs according to the levels of proteinuria (proteinuria $<3.5 \mathrm{~g} / 24$ hours, $\mathrm{n}=23$; proteinuria $\geq 3.5 \mathrm{~g} / 24$ hours, $\mathrm{n}=43)$. The numbers of urinary MPs $\left(\times 10^{6} / \mathrm{mL}\right)$ were shown as medians and IQRs. CKD, chronic kidney disease; MPs, microparticles; HCs, healthy controls; IMN, idiopathic membranous nephropathy; IgAN, IgA nephropathy; LN, lupus nephritis; DN, diabetic nephropathy; IQRs, interquartile ranges. 

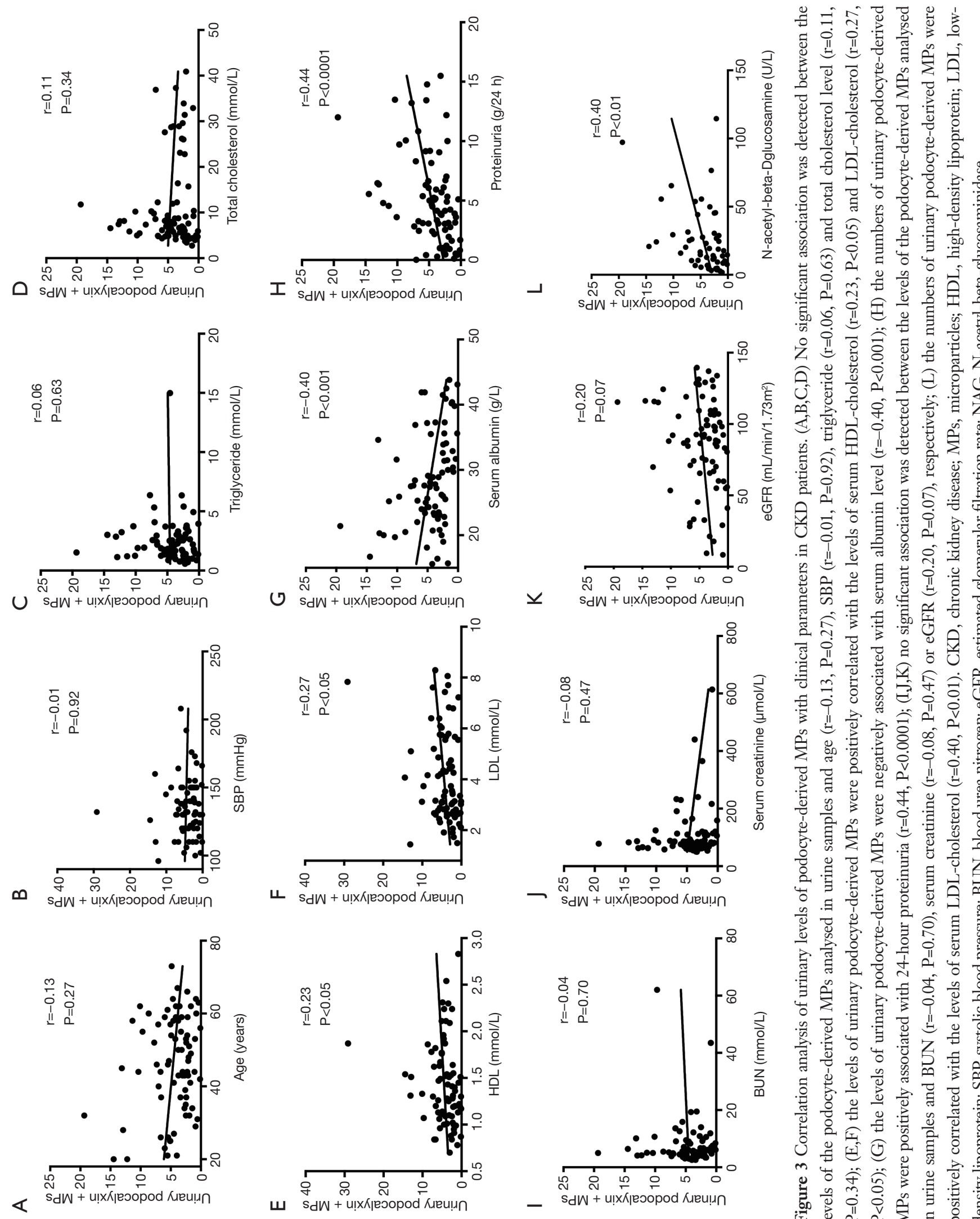

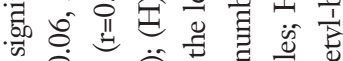
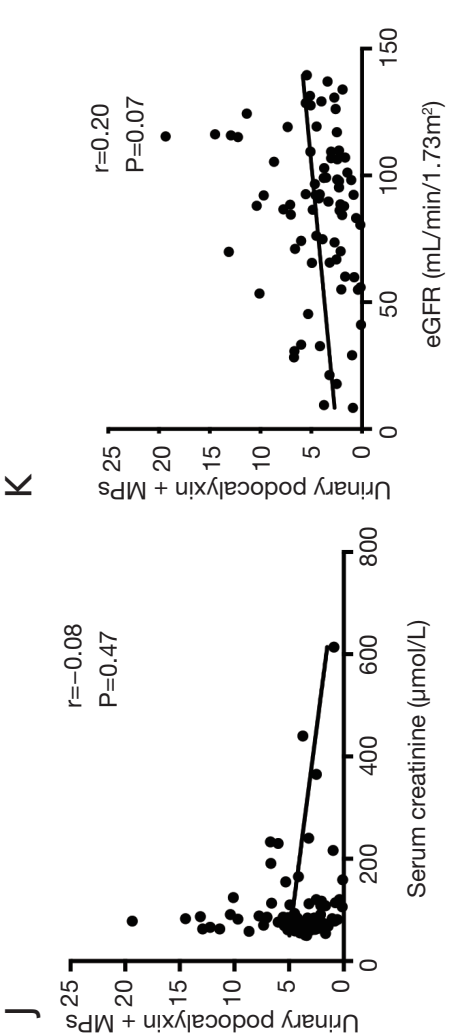

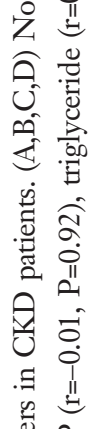
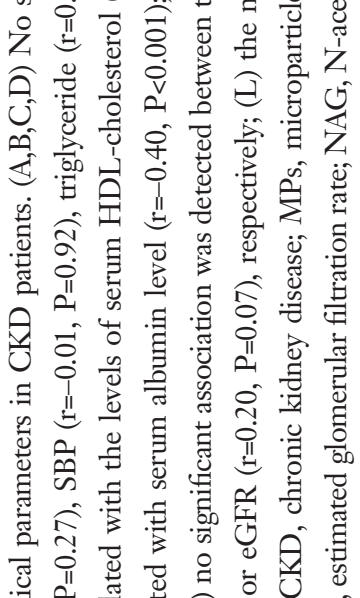

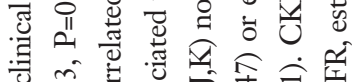

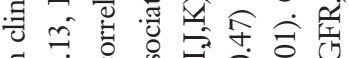

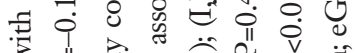

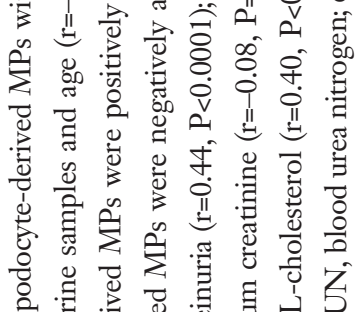

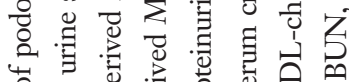

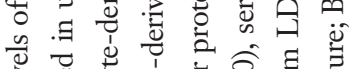

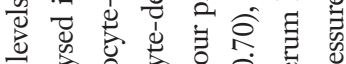

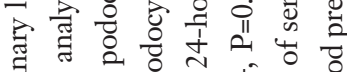

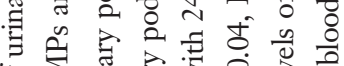

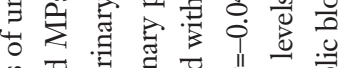

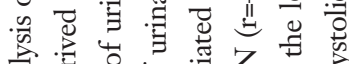

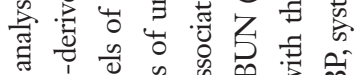

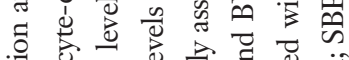

.

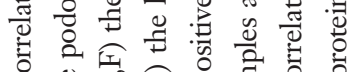

نे

m

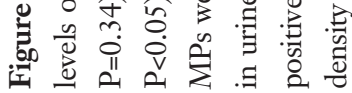


A

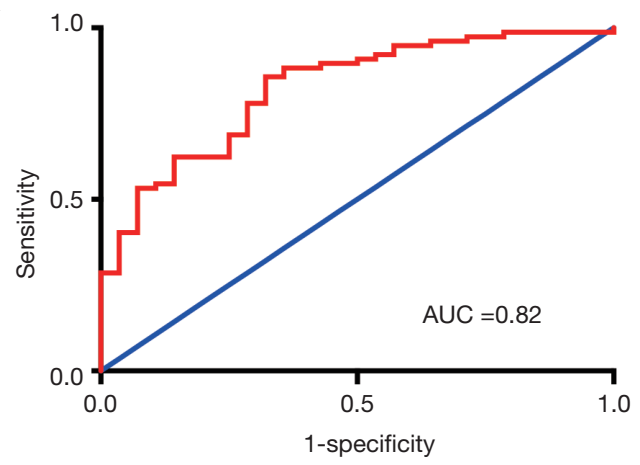

B

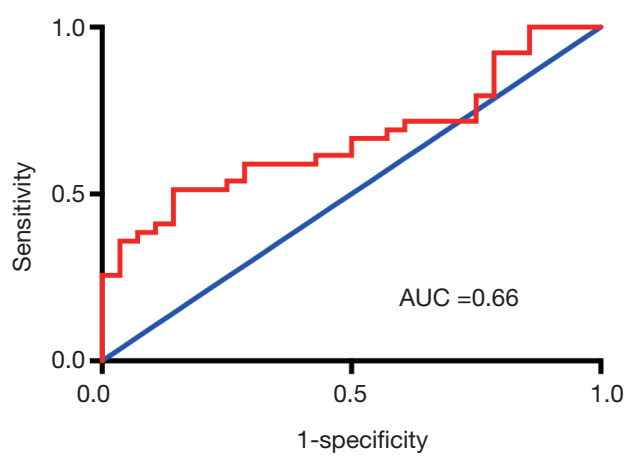

Figure 4 ROC curves were calculated to assess the discrimination power for urinary levels of podocyte-derived MPs. (A) Urinary levels of podocyte-derived MPs differentiated CKD patients with HCs (AUC, 0.82; 95\% CI 0.74-0.91; P<0.01) (13,14); (B) urinary levels of podocyte-derived MPs differentiated CKD patients with global glomerulosclerosis and without global glomerulosclerosis (AUC, $0.66 ; 95 \%$ CI 0.52-0.79; $\mathrm{P}<0.05)$. ROC, receiver-operating characteristic; CKD, chronic kidney disease; MPs, microparticles; HCs, healthy controls; AUC, area under curve; CI, confidence interval.

Table 3 Spearman correlation coefficients between global glomerulosclerosis, urinary levels of podocyte-derived MPs, and selected parameters in CKD patients

\begin{tabular}{lcc}
\hline & & Global glomerulosclerosis (\%) \\
\cline { 2 - 3 } Parameter & $r$ & P value \\
\hline Age (years) & 0.23 & 0.07 \\
SBP $(\mathrm{mmHg})$ & 0.38 & $<0.01$ \\
DBP $(\mathrm{mmHg})$ & 0.25 & 0.051 \\
Fast glucose (mmol/L) & 0.34 & $<0.05$ \\
Total cholesterol (mmol/L) & -0.33 & $<0.01$ \\
Triglyceride (mmol/L) & -0.05 & 0.72 \\
BUN (mmol/L) & 0.39 & $<0.01$ \\
Serum creatinine $(\mu \mathrm{mol} / \mathrm{L})$ & 0.40 & 0.001 \\
Proteinuria $(\mathrm{g} / 24$ hours) & -0.24 & 0.051 \\
eGFR (mL/min per $\left.1.73 \mathrm{~m}^{2}\right)$ & -0.48 & $<0.001$ \\
Urinary podocyte-derived MPs & -0.26 & $<0.05$
\end{tabular}

CKD, chronic kidney disease; MPs, microparticles; SBP, systolic blood pressure; DBP, diastolic blood pressure; BUN, blood urea nitrogen; eGFR, estimated glomerular filtration rate.

of podocalyxin protein checked by ELISA. In glomerular diseases with severe podocyte damage, urinary-podocalyxin (u-PCX) reflected the microvilli shedding process. High levels of u-PCX could be considered to reflect marked microvillous transformation and vesicular shedding. Imaizumi et al. also demonstrated that the urinary level of podocalyxin is a candidate marker for $\mathrm{MN}$ and it can be applied for the diagnostic models in combination with other clinical parameters (18). The excretion of podocalyxin positive elements is correlated with the degree of glomerular destruction. Podocyte membrane vesicles in urine originate from tip vesiculation of podocyte microvilli (19). As to the certain levels of podocyte-derived MPs in HCs, to some extent, a certain number of MPs might have been shed by podocyte microvilli into the urine due to normal physiological turnover, since microvillous transformation is 
A

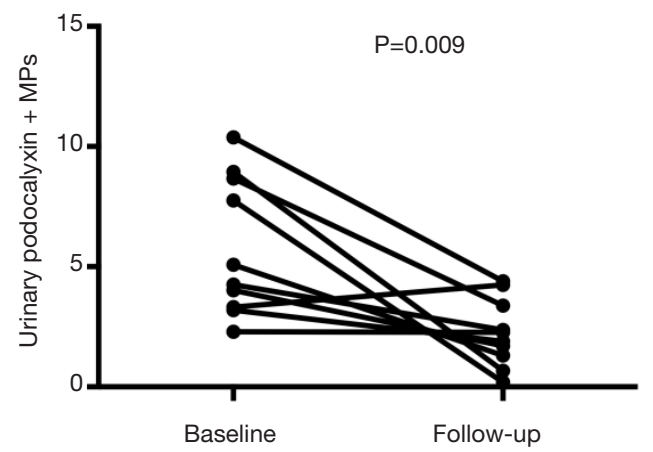

B

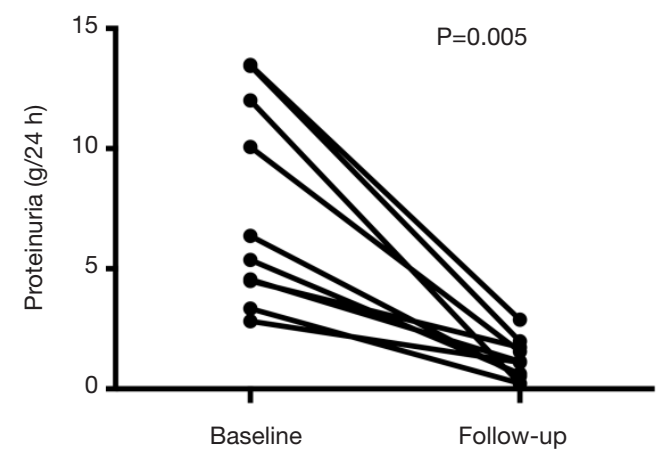

Figure 5 Effect of immunosuppressive therapy on urinary levels of podocyte-derived MPs in IMN patients. (A) Combined treatment of prednisone with tacrolimus in IMN patients, which induced partial remission (five patients) or complete remission (five patients), resulting in significant decreased urinary levels of podocyte-derived MPs and proteinuria; (B) the urinary levels of podocyte-derived MPs were determined by flow cytometry in ten IMN patients before and after six-month immunosuppressive therapy. IMN, idiopathic membranous nephropathy; MPs, microparticles.

occasionally found on the normal glomerulus.

Our results showed that there was a significant positive correlation between urinary podocyte-derived MPs levels and 24-hour proteinuria level. Proteinuria is a sign of GFB damage and an independent risk factor for glomerular diseases. The degree of proteinuria associates with the deterioration speed of CKD. Dyslipidemia is one of indicative manifestations of nephrotic syndrome, as urinary protein loss would stimulate an increase of LDL synthesis by the liver. Likewise, in the case of hypercholesterolemia, podocyte injury induces glomerular lipid accumulation, and further exacerbate glomerular lesions (20). The positive correlation between podocyte-derived MPs and HDL/ LDL-cholesterol concentration would provide new angle to understand interaction between podocyte injury and dyslipidemia in CKD patients, especially in those with massive proteinuria. In contrast, the serum creatinine level or eGFR did not correlate with urinary podocytederived MPs levels. Change in serum creatinine levels and eGFR typically reflects declined renal function only in the advanced stage of the kidney disease when functioning nephrons are lost, not in the earlier stages. Since the presence of urinary podocyte-derived MPs reflects podocyte injury in the relatively earlier stages of kidney disease, the lack of correlation between serum creatinine/eGFR and urinary podocyte-derived MPs levels is not hard to explain. Hypertensive or ischemic kidney disease might induce podocyte damage. Previous studies have shown that urinary podocyte-derived EVs levels were increased in patients with renovascular hypertension compared with healthy volunteers and patients with essential hypertension (11). Accordingly, the levels of urinary podocyte derived MPs in CKD patients treated with ARB or ACE inhibitors were decreased. However, no significant correlation was observed between urinary podocyte-derived MPs levels and SBP.

A main finding of our study is the difference of urinary podocyte-derived MPs levels depended on the degree of glomerulosclerosis. The urinary levels of podocytederived MPs were markedly lower in CKD patients with glomerulosclerosis compared with that in those without glomerulosclerosis. There was a significant negative association between urinary podocyte-derived MP and the percentage of glomerulosclerosis. This suggests that urinary podocyte-derived MP is a good biomarker for the diagnosis of glomerulosclerosis, and the number of podocyte-derived MPs in urine possibly represents the degree of glomerulosclerosis. Podocytes, one of critical cell components of the GFB, belong to highly differentiated cells and are almost non-renewable. Persistent podocyte injuries cause glomerular hypertrophy, mesangial matrix deposition, vessel occlusion, which are common pathological change of glomerulosclerosis from focal segmental to diffuse distribution (6). Previous studies demonstrated the lack of podocytes is associated with the occurrence of glomerulosclerosis. If the cells are reduced by $10 \%$ to $20 \%$, then glomerulosclerosis is beginning (21). Thus, the loss of podocytes in situ might be an explanation to the decline of MP shedding.

Moreover, MPs are supposed to be interact with neighbouring or distant cells by transferring bioactive 
proteins, receptors, and mRNA $(22,23)$. From our results, the levels of podocyte-derived MPs were significantly correlated with urinary NAG, while there was no difference for podocyte-derived MPs levels in CKD patients with different degree of tubulointerstitial lesions. NAG is a proximal tubular damage marker. It was reported that in proteinuric glomerular diseases, increased NAG excretion occurs even in the absence of morphological evidence of tubular cell damage. The increased uptake of filtered proteins leads to increased lysosomal activity of tubular epithelial cells (24). It is reported that podocyte-derived MPs induced tubular epithelial cell fibrotic responses via $\mathrm{p} 38$ MAPK and CD36 in vitro, which suggests that such interaction may contribute to the development of tubular fibrosis (25). The discrepancy may due to complex pathophysiological conditions in vivo, and somehow the semi-quantitative assessments cannot completely reflect the cytological status of renal tubular interstitial damage. Further research is needed to prove our speculation that early podocyte-derived MPs shedding may provide a novel mechanic interaction between glomerular podocyte injury and tubular dysfunction.

MPs have been reported to reflect ongoing and potentially reversible cell stress, which could help to identify at-risk podocytes before irreversible cellular injury/death. To further investigate whether urinary levels of podocyte-derived MPs reflect the degree of podocyte injury, we treated IMN patients with prednisone plus tacrolimus. Results showed that after treatment, proteinuria and the urinary levels of podocyte-derived MPs in IMN patients were distinctly reduced, indicating that monitoring levels of urinary levels of podocytederived MPs could indicate therapy efficacy and remission of disease. Ten IMN patients among all participants were followed up for six months. The short-term remission rate was very optimistic, five patients achieved part remission (proteinuria $>0.5 \mathrm{~g} / 24$ hours but less than half of the baseline), the other five patients acquired complete remission (proteinuria $<0.5 \mathrm{~g} / 24$ hours). It was reported that tacrolimus protected podocytes from injuries through stabilizing the podocyte cytoskeleton and inhibiting apoptosis (26).

In conclusion, the urinary levels of podocyte-derived MPs were closely associated with podocyte injury and glomerulosclerosis. The change of urinary podocyte-derived MPs levels could be useful for monitoring disease activity in CKD patients, which might be a non-invasive biomarker for the evaluation of early CKD progression.

\section{Limitation}

Firstly, the number of IMN patients with prospective follow-up was too small. Secondly, the present study cannot link higher podocyte-derived MP counts to the prognosis of CKD, which would require more longitudinal studies to clarify its value as a biomarker.

\section{Acknowledgments}

Funding: This work was supported by the Jiangsu Province Social Development Project (BE2018744), the Project for Jiangsu Provincial Medical Talent (ZDRCA2016077), the Jiangsu Province Six Talent Peaks Project (2015-WSN002), the Fundamental Research Funds for the Central Universities (KYCX18-0182, KYCX17-0169, KYZZ150061), and the Jiangsu Province Ordinary University Graduate Research Innovation Project (SJZZ16-004). Joint Project of Southeast University and Pharmaceutical University of China (2242019K3DZ03).

\section{Footnote}

Conflicts of Interest: The authors have no conflicts of interest to declare.

Ethical Statement: The authors are accountable for all aspects of the work in ensuring that questions related to the accuracy or integrity of any part of the work are appropriately investigated and resolved. All the patients were written informed consent to participate in this study, and the study protocol was approved by the clinical research ethics committee of Zhongda Hospital affiliated to Southeast University (No. 2016ZDSYLL003.0). The study was conducted in adherence to the Declaration of Helsinki.

\section{References}

1. Romagnani P, Remuzzi G, Glassock R, et al. Chronic kidney disease. Nat Rev Dis Primers 2017;3:17088.

2. Kriz W, Lemley KV. A potential role for mechanical forces in the detachment of podocytes and the progression of CKD. J Am Soc Nephrol 2015;26:258-69.

3. Neal CR, Muston PR, Njegovan D, et al. Glomerular filtration into the subpodocyte space is highly restricted under physiological perfusion conditions. Am J Physiol Renal Physiol 2007;293:F1787-98.

4. Liu $\mathrm{Y}$, Hitomi H, Diah $\mathrm{S}$, et al. Roles of $\mathrm{Na}^{+} / \mathrm{H}^{+}$exchanger 
type 1 and intracellular $\mathrm{pH}$ in angiotensin II-induced reactive oxygen species generation and podocyte apoptosis. J Pharmacol Sci 2013;122:176-83.

5. Bruggeman LA, Drawz PE, Kahoud N, et al. TNFR2 interposes the proliferative and NF- $\mathrm{\kappa B}$-mediated inflammatory response by podocytes to TNF- $\alpha$. Lab Invest 2011;91:413-25.

6. Nagata M. Podocyte injury and its consequences. Kidney Int 2016;89:1221-30.

7. Karpman D, Ståhl AL, Arvidsson I. Extracellular vesicles in renal disease. Nat Rev Nephrol 2017;13:545-62.

8. Burger D, Schock S, Thompson CS, et al. Microparticles: biomarkers and beyond. Clin Sci (Lond) 2013;124:423-41.

9. Souza AC, Yuen PS, Star RA. Microparticles: markers and mediators of sepsis-induced microvascular dysfunction, immunosuppression, and AKI. Kidney Int 2015;87:1100-8.

10. Burger D, Thibodeau JF, Holterman CE, et al. Urinary podocyte microparticles identify prealbuminuric diabetic glomerular injury. J Am Soc Nephrol 2014;25:1401-7.

11. Kwon SH, Woollard JR, Saad A, et al. Elevated urinary podocyte-derived extracellular microvesicles in renovascular hypertensive patients. Nephrol Dial Transplant 2017;32:800-7.

12. Zhou H, Yuen PS, Pisitkun T, et al. Collection, storage, preservation, and normalization of human urinary exosomes for biomarker discovery. Kidney Int 2006;69:1471-6.

13. Doyonnas R, Nielsen JS, Chelliah S, et al. Podocalyxin is a CD34-related marker of murine hematopoietic stem cells and embryonic erythroid cells. Blood 2005;105:4170-8.

14. Sassetti C, Tangemann K, Singer MS, et al. Identification of podocalyxin-like protein as a high endothelial venule ligand for L-selectin: parallels to CD34. J Exp Med 1998;187:1965-75.

15. Vitureira N, McNagny K, Soriano E, et al. Pattern of expression of the podocalyxin gene in the mouse brain during development. Gene Expr Patterns 2005;5:349-54.

16. Sekulic M, Pichler Sekulic S. A compendium of urinary biomarkers indicative of glomerular podocytopathy. Patholog Res Int 2013;2013:782395.

Cite this article as: $\mathrm{Lu} \mathrm{J}, \mathrm{Hu} Z \mathrm{ZB}$, Chen PP, Lu CC, Zhang JX, Li XQ, Yuan BY, Huang SJ, Ma KL. Urinary levels of podocytederived microparticles are associated with the progression of chronic kidney disease. Ann Transl Med 2019;7(18):445. doi: 10.21037/atm.2019.08.78
17. Hara M, Yamagata K, Tomino Y, et al. Urinary podocalyxin is an early marker for podocyte injury in patients with diabetes: establishment of a highly sensitive ELISA to detect urinary podocalyxin. Diabetologia 2012;55:2913-9.

18. Imaizumi $T$, Nakatochi $M$, Akiyama $S$, et al. Urinary podocalyxin as a biomarker to diagnose membranous nephropathy. PLoS One 2016;11:e0163507.

19. Hara M, Yanagihara T, Hirayama Y, et al. Podocyte membrane vesicles in urine originate from tip vesiculation of podocyte microvilli. Hum Pathol 2010;41:1265-75.

20. Hara S, Kobayashi N, Sakamoto K, et al. Podocyte injury-driven lipid peroxidation accelerates the infiltration of glomerular foam cells in focal segmental glomerulosclerosis. Am J Pathol 2015;185:2118-31.

21. Shen P, Shen J, Li W, et al. Urinary podocyte can be an indicator for the pathogenetic condition of patients with IgA nephropathy. Clin Lab 2014;60:1709-15.

22. Ranghino A, Dimuccio V, Papadimitriou E, et al. Extracellular vesicles in the urine: markers and mediators of tissue damage and regeneration. Clin Kidney J 2015;8:23-30.

23. Fernandez-Martínez AB, Torija AV, Carracedo J, et al. Microparticles released by vascular endothelial cells increase hypoxia inducible factor expression in human proximal tubular HK-2 cells. Int J Biochem Cell Biol 2014;53:334-42.

24. Navarro JF, Mora C, Muros M, et al. Effects of pentoxifylline administration on urinary $\mathrm{N}$-acetyl-betaglucosaminidase excretion in type 2 diabetic patients: a short-term, prospective, randomized study. Am J Kidney Dis 2003;42:264-70.

25. Munkonda MN, Akbari S, Landry C, et al. Podocytederived microparticles promote proximal tubule fibrotic signaling via p38 MAPK and CD36. J Extracell Vesicles 2018;7:1432206.

26. Liao R, Liu Q, Zheng Z, et al. Tacrolimus protects podocytes from injury in lupus nephritis partly by stabilizing the cytoskeleton and inhibiting podocyte apoptosis. PLoS One 2015;10:e132724. 